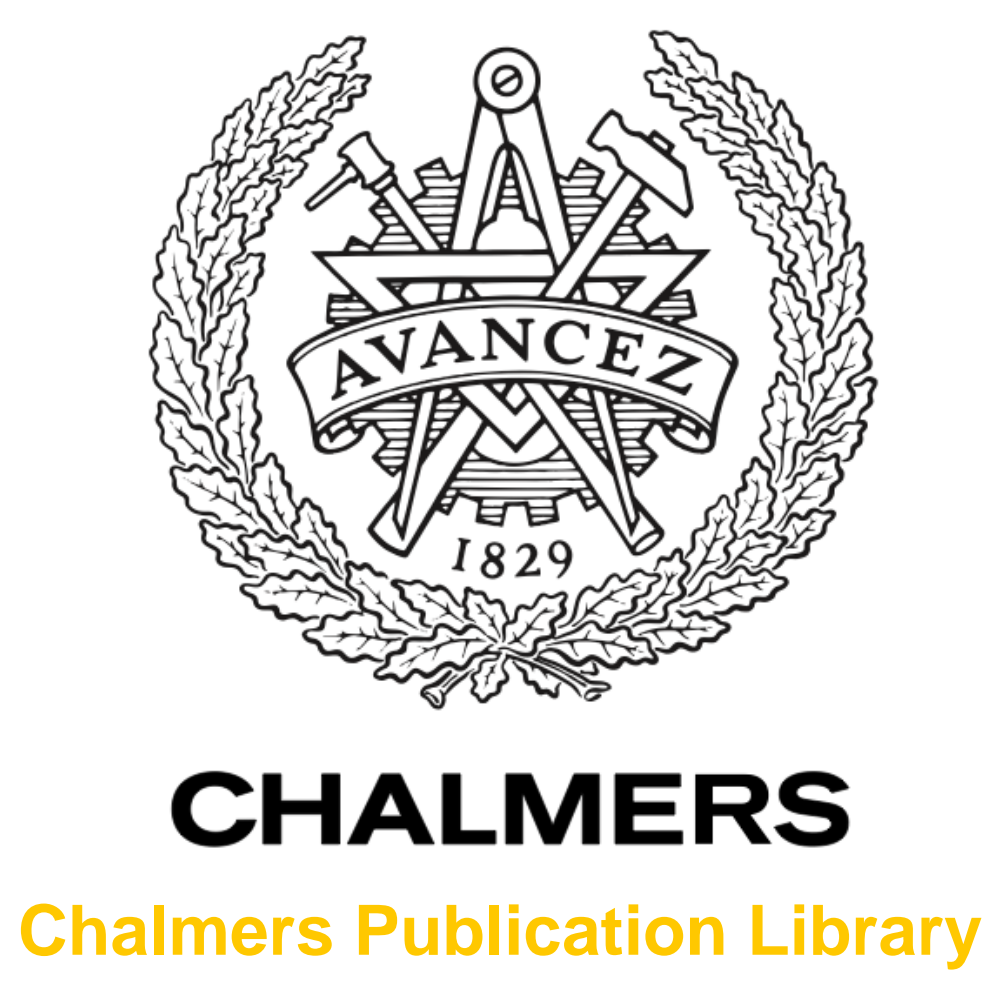

\title{
Improved MIMO Throughput With Inverse Power Allocation - Study Using USRP Measurement in Reverberation Chamber
}

This document has been downloaded from Chalmers Publication Library (CPL). It is the author's version of a work that was accepted for publication in:

IEEE Antennas and Wireless Propagation Letters (ISSN: 1536-1225)

Citation for the published paper:

Chen, X. ; Einarsson, B. ; Kildal, P. (2014) "Improved MIMO Throughput With Inverse Power Allocation - Study Using USRP Measurement in Reverberation Chamber". IEEE Antennas and Wireless Propagation Letters, vol. 13 pp. 1494-1496.

Downloaded from: http://publications.lib.chalmers.se/publication/201863

Notice: Changes introduced as a result of publishing processes such as copy-editing and formatting may not be reflected in this document. For a definitive version of this work, please refer to the published source. Please note that access to the published version might require a subscription. 


\title{
Improved MIMO Throughput With Inverse Power Allocation - Study Using USRP Measurement in Reverberation Chamber
}

\author{
Xiaoming Chen, Bjarni bór Einarsson, and Per-Simon Kildal, Fellow, IEEE
}

\begin{abstract}
In this letter, we study the spatial multiplexing throughputs of multiple-input-multiple-output (MIMO) systems with fixed modulation and coding scheme (i.e., fixed peak rate) based on measurement of the universal software radio peripheral (USRP) in a reverberation chamber. In particular, we propose a simple inverse power allocation scheme for the singular value decomposition (SVD)-based MIMO system. Using the proposed power allocation, the power saving (for achieving the same throughput), compared to the SVD-MIMO without power allocation, is about $2.5 \mathrm{~dB}$. Good agreement is observed between the USRP measurement and the simulation.
\end{abstract}

Index Terms-Multiple-input-multiple-output (MIMO), reverberation chamber (RC), universal software radio peripheral (USRP).

\section{INTRODUCTION}

M EASUREMENT-BASED characterizations of multiple-input-multiple-output (MIMO) systems are essential for the development of MIMO devices. Being a convenient tool for emulating multipath environments, the reverberation chamber (RC) has been used for various over-the-air (OTA) tests of MIMO systems [1]-[6]. In particular, simple yet accurate throughput models have been presented in [1]-[3], which have been validated by measurements of Long Term Evolution (LTE) systems in an RC.

In this letter, we resort to the universal software radio peripheral (USRP). USRP is an inexpensive and versatile platform that allows implementations of various algorithms for telecommunications [7]. Thus, unlike the previous RC-based MIMO OTA studies that are limited to the standards of LTE [1]-[4] or wireless local area network (WLAN) [5], we have more flexibility and can examine different MIMO algorithms when using the USRP. In the present work, we focus on the spatial multiplexing throughput. Specifically, we compare the throughput performance of the singular value decomposition (SVD)-based MIMO with/without power allocation and that of the zero-forcing (ZF)-based MIMO. For simplicity and due to

Manuscript received May 17, 2014; accepted July 21, 2014. Date of publication July 25, 2014; date of current version August 08, 2014. This work was supported in part by The Swedish Governmental Agency for Innovation Systems (VINNOVA) within the VINN Excellence Center Chase.

The authors are with the Department of Signals and Systems, Chalmers University of Technology, 41296 Gothenburg, Sweden (e-mail: xiaoming.chen@chalmers.se).

Color versions of one or more of the figures in this letter are available online at http://ieeexplore.ieee.org.

Digital Object Identifier 10.1109/LAWP.2014.2342217 the OTA testing constraints, we constructed a fixed peak rate $2 \times 2$ MIMO system using the USRP, where the probability of detecting both data streams is limited to the probability of detecting the worst stream. The purpose is to verify by measurements the theoretical advantage of a simple inverse power allocation scheme for the SVD case.

\section{THEORY}

We use the throughput model presented in [1] and [3] for diversity and spatial multiplexing, respectively, based on an ideal threshold receiver. For the sake of completeness of this letter, we briefly repeat the model here. The average package error rate (PER) in a fading channel can be approximated as [1]

$$
\overline{P_{e}}(\bar{\gamma})=\int_{0}^{\gamma_{\mathrm{th}}} f(\gamma ; \bar{\gamma}) d \gamma=F\left(\gamma_{\mathrm{th}} ; \bar{\gamma}\right)
$$

where $\gamma$ is the instantaneous signal-to-noise ratio (SNR), $\bar{\gamma}$ represents the average $\gamma, \gamma_{\text {th }}$ is the threshold value, and $f$ and $F$ denote the probability density function (PDF) and cumulative distribution function (CDF) of $\gamma$ for a given $\bar{\gamma}$, respectively. The throughput is then [1]

$$
T_{\text {put }}(\bar{\gamma})=T_{\text {put,max }}\left(1-F\left(\gamma_{\text {th }} / \bar{\gamma}\right)\right)
$$

where $T_{\text {put,max }}$ denotes the maximum data rate. Defining the relative throughput as $T_{\text {put }} / T_{\text {put,max }}$, it can be seen from (2) that the relative throughput is the same as the probability of detection (PoD) $\left(1-F\left(\gamma_{\text {th }} / \bar{\gamma}\right)\right)$.

In a flat fading channel, the MIMO system can be modeled as

$$
\mathbf{y}=\mathbf{H} \mathbf{x}+\mathbf{n}
$$

where $\mathbf{H}$ is the MIMO channel matrix, $\mathbf{x}$ and $\mathbf{y}$ are the transmitted and received signal vectors, respectively, and $\mathbf{n}$ is the noise vector with independent identically distributed (i.i.d.) Gaussian variables. Note that, for an orthogonal frequency-division multiplexing (OFDM) system in a frequency-selective and quasi-static fading channel, the model (2) can be regarded as one subcarrier of the MIMO-OFDM system, and the frequency selectivity can be modeled by coherently combining the independent subcarriers.

The receiver usually has access to the channel state information (CSI) via channel estimation and tracking. In a time-division duplex (TDD) system, the transmitter can obtain the CSI by 
exploiting the channel reciprocity (assuming the channel stays the same in the adjacent downlink and uplink time-slots). When CSI exists at both MIMO ends, it is natural to use the SVD-based MIMO configuration [8].

Let the SVD of $\mathbf{H}$ be $\mathbf{H}=\mathbf{U} \boldsymbol{\Lambda} \mathbf{V}^{H}$, where $\mathbf{U}$ and $\mathbf{V}$ are the unitary matrices, and $\boldsymbol{\Lambda}$ is a diagonal matrix consisting of the singular values of $\mathbf{H}$. Then, the precoding and power allocation is done by multiplying the signal vector $\mathbf{s}$ by $\mathbf{V P}$, i.e., $\mathbf{x}=$ VPs, where $\mathbf{P}$ is a diagonal matrix whose elements correspond to the allocated power for each stream. The decoding is done by multiplying $\mathbf{y}$ by $\mathbf{U}^{H}, \mathrm{r}=\mathbf{U}^{H} \mathbf{y}$. The resulting (interferencefree) parallel MIMO channel is then

$$
\mathbf{r}=\mathbf{\Lambda} \mathbf{P s}+\mathbf{z}
$$

where $\mathbf{z}=\mathbf{U}^{H} \mathbf{n}$. Note that $\mathbf{s}$ and $\mathbf{z}$ have the same statistics as $\mathbf{x}$ and $\mathbf{n}$, respectively, because $\mathbf{V}$ and $\mathbf{U}$ are unitary matrices.

Note that the well-known water-filling algorithm [8] for optimally allocating power (i.e., determining optimal $\mathbf{P}$ ) is derived from the information capacity assuming each spatial stream can support any maximum rate. However, in practice, the available modulation and coding schemes (MCSs) are finite, implying that only a finite number of peak rates can be supported. In this case, the water-filling technique is not optimal anymore. In the present work, we propose instead an inverse power allocation scheme: to allocate more power to the weaker eigen-channel and less power to the stronger one so that the received SNRs of all the streams are the same. This is done in order to maximize the PoD of receiving all bit streams. Mathematically, the power allocation matrix $\mathbf{P}$ can be expressed as

$$
\mathbf{P}=\mu \boldsymbol{\Lambda}^{-1}
$$

where the parameter $\mu$ is a constant making sure that the total power is the same before and after the precoding and power allocation. Denoting VP as $\mathbf{F}$

$$
N_{t}=\operatorname{tr}\left[\mathbf{F}^{H} \mathbf{F}\right]=\operatorname{tr}\left[\mathbf{P}^{H} \mathbf{V}^{H} \mathbf{V P}\right]=\operatorname{tr}\left[\mathbf{P}^{2}\right]=\mu^{2} \operatorname{tr}\left[\mathbf{\Lambda}^{-2}\right] .
$$

Thus

$$
\mu=\sqrt{N_{\mathrm{t}} / \operatorname{tr}\left[\boldsymbol{\Lambda}^{-2}\right]}
$$

where $N_{\mathrm{t}}$ is the number of transmitting antennas. We refer to the power allocation scheme (5) as an inverse power allocation. This makes when we want to improve the PoD of all streams (i.e., the relative maximum throughput) because this corresponds to the PoD of detecting the worst stream.

If no power allocation is applied, the transmitted power is divided equally between all streams, i.e., $\mathbf{P}=\mathbf{I}$. In Section III, we will show how much we can gain by using the inverse power allocation.

The SVD-MIMO scheme accounts for the situation when the CSI is known at both MIMO ends. In case the CSI is known only at the receiving side, we can apply the ZF equalization [8]. The ZF-based MIMO throughput model was presented in [3]. For the sake of conciseness, we will not repeat it here. Nevertheless, both simulation and measurement of the ZF-based MIMO throughput will be presented in Section III for comparison to the SVD-based MIMO throughputs.
We will now show that by combining precoding $\mathbf{W}=\mathbf{V}$ with $\mathrm{ZF}$, we can achieve the same performance as that which we have with the SVD-based MIMO system.

Proof: A $2 \times 2$ MIMO system with a precoder $\mathbf{W}$ (in a flat-fading channel) can be modeled as

$$
\mathbf{y}=\mathbf{H W} \mathbf{s}+\mathbf{n}=\mathbf{U} \boldsymbol{\Lambda} \mathbf{V}^{H} \mathbf{W} \mathbf{s}+\mathbf{n} .
$$

Since $\mathbf{V}$ and $\mathbf{W}$ are unitary matrices, one can choose $\mathbf{W}$ such that

$$
\mathbf{V}_{\text {eq }}=\mathbf{V}^{H} \mathbf{W}=\left[\begin{array}{cc}
\cos \theta & -\sin \theta \\
\sin \theta & \cos \theta
\end{array}\right]
$$

where $\theta \in[0, \pi / 2]$. The SNR of the $2 \times 2$ MIMO system with precoding $\mathbf{W}$ and $\mathrm{ZF}$ receiver is

$$
\gamma_{i}=\frac{1}{\left[\left(\left(\mathbf{H} \mathbf{V}_{\mathrm{eq}}\right)^{H} \mathbf{H} \mathbf{V}_{\mathrm{eq}}\right)^{-1}\right]_{i, i}}=\frac{1}{\frac{\cos ^{2} \theta}{\lambda_{i}}+\frac{\sin ^{2} \theta}{\lambda_{l}}}
$$

where $i, l \in\{1,2\}, i \neq l$, and $\lambda_{i}$ is the $i$ th diagonal element of $\boldsymbol{\Lambda}$. Letting $\theta=0$ (i.e., $\mathbf{W}=\mathbf{V}$ ), (10) reduces to $\gamma_{i}=\lambda_{i}^{2}$ (i.e., the SNR of the SVD-based MIMO system).

This observation is rather intuitive. When $\theta=0, \mathbf{V}^{H} \mathbf{W}$ becomes identity matrix, i.e., the precoder $\mathbf{W}$ diagonalizes the transmitting-side singular vector matrix; and the receiving-side singular vector matrix can be diagonalized similarly by a linear ZF decoder. Thus, parallel channels identical to the SVD case are obtained. This proof indicates that the $\mathrm{ZF}$ equalizer is equally good as the decoding matrix of the SVD-MIMO. Therefore, the performance difference of the ZF- and SVD-based MIMO systems is only due to the fact that the (open-loop) ZF-based MIMO system does not have CSI at the transmitting side.

\section{MeAsurements AND Results}

LabVIEW [9] is used in this work to control the USRP. The sampling rate is set to $400 \mathrm{kHz}$, and each symbol is selected as eight samples, resulting in a symbol rate of $50 \mathrm{k} \mathrm{symbols} / \mathrm{s}$. This allows for a maximum bit rate of $100 \mathrm{~kb} / \mathrm{s}$ with gray coded quadrature phase shift keying (QPSK). However, due to a large packet overhead, the achievable bit rate is only $33 \mathrm{~kb} / \mathrm{s}$. For the operating frequency $915 \mathrm{MHz}$, with a root raised cosine as the pulse shaping filter with a roll-off factor of 0.5 and a length of 6 , the system bandwidth is $75 \mathrm{kHz}$, which is much smaller than the $3.5-\mathrm{MHz}$ coherence bandwidth (corresponding to a quality factor of 261) for the measurement setup in the RC. The used $\mathrm{RC}$ has a size of $1.75 \times 1.80 \times 1.25 \mathrm{~m}^{3}$ and is equipped with two translating plate stirrers with sizes of $0.97 \times 0.40 \mathrm{~m}^{2}$ and $0.88 \times 0.30 \mathrm{~m}^{2}$, respectively, and a turntable platform with a diameter of about $0.6 \mathrm{~cm}$ (see [10] for a detailed description of it). Both transmitting and receiving antennas are wideband triangular-shaped monopole antennas that are orthogonally polarized with sufficient separation to ensure no correlation. The stirrers in the RC run stepwise to 300 positions, and at each stirrer position, 200 packets are sent. The measurements are performed over the power range of -60 to $-90 \mathrm{dBm}$.

Fig. 1 shows the measurement setup of conductive and OTA measurements. Fig. 2 (top) shows the conductive throughput of 


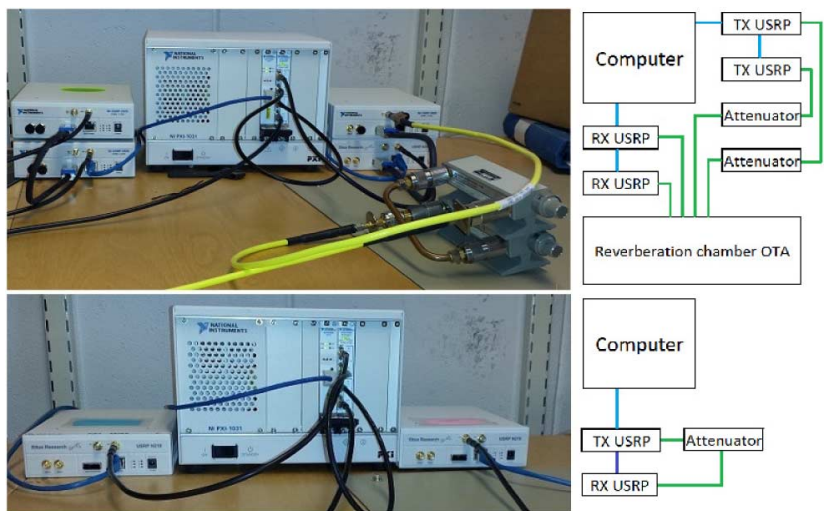

Fig. 1. (top) Conductive and (bottom) OTA measurement setup.
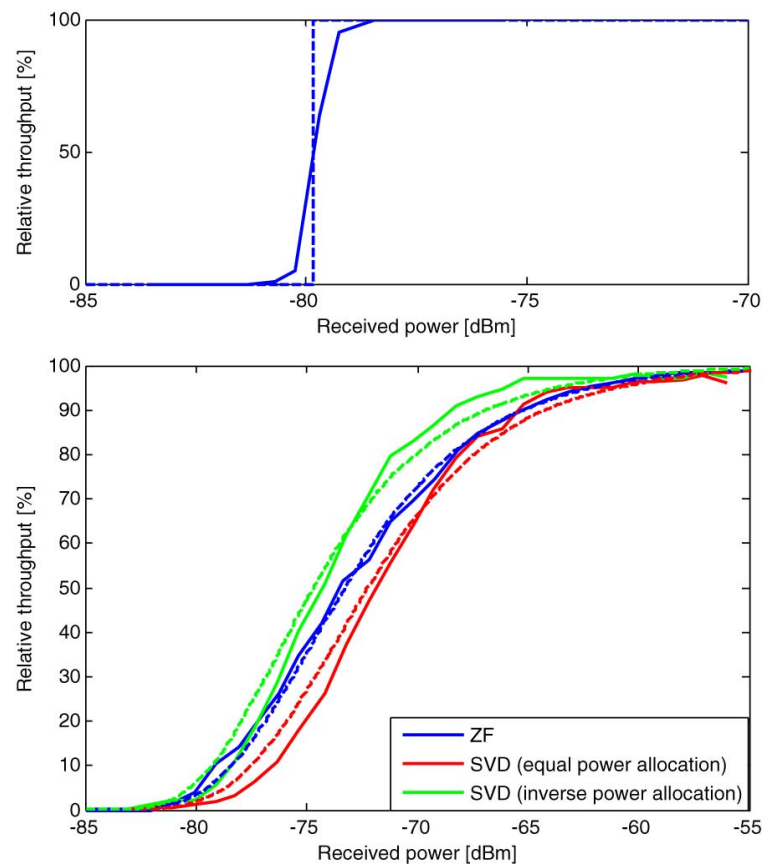

Fig. 2. (top) Conductive USRP throughput and (bottom) OTA throughputs of $\mathrm{ZF}$ - and SVD-based $2 \times 2$ MIMO system with and without inverse power allocation. Solid curve represents measured, and dashed curve simulated results.

the USRP. The threshold value of $-79.8 \mathrm{dBm}$ is read out from the conductive measurement at $50 \%$ throughput level. Note that the noise power is not available from the measurement, so all the throughput results in this section are presented as a function of received power instead of SNR. Since the noise power is canceled out by $\gamma_{\text {th }} / \bar{\gamma}$ in the throughput model (2), there is no loss of generality in doing so. The measurements in the reverberation chamber are accurate to a few tenths of a decibel [10].

Fig. 2 (bottom) shows the relative throughputs of the SVD-based $2 \times 2$ MIMO systems both with and without the inverse power allocation. As a reference, we also plot the relative throughput of the $2 \times 2$ (open-loop) ZF-based MIMO system in the same figure. The solid curves in Fig. 2 represent measured throughput, and the dashed curves correspond to the simulated throughput using the throughput model. There is good agreement between measurements and simulations.
Furthermore, we see that, without the inverse power allocation, the SVD-based MIMO throughput for a fixed MCS is even slightly worse than that of the open-loop ZF-based MIMO. The reason is that with SVD, the best eigen-channel is better than for the ZF-case, but the worst eigen-channel is worse (and the probability of detecting two streams equals that of detecting the worst one for the fixed peak rate system). Note that since the water-filling technique puts more power on the best eigen-channel and less on the worst one, the relative throughput of an SVD-based MIMO system with water-filling technique will be even worse than the equal power allocation case. Therefore, the water-filling technique is not used in practical systems such as LTE [11]. We see from Fig. 2(b) that the SVD-based MIMO with the inverse power allocation is about $2.5 \mathrm{~dB}$ better (in terms of power cost) than that without power allocation and about $1.5 \mathrm{~dB}$ better than the open-loop ZF-based MIMO system.

\section{CONCLUSION}

In this letter, the throughputs of ZF- and SVD-based MIMO systems are studied by measuring the USRP in an RC. An inverse power allocation scheme for the fixed-peak-rate SVD-MIMO systems is proposed. The SVD-based MIMO with the inverse power allocation is about $2.5 \mathrm{~dB}$ better than that without power allocation, and about $1.5 \mathrm{~dB}$ better than the open-loop ZF-based MIMO system. There is good agreement between measurements and simulations.

\section{REFERENCES}

[1] P.-S. Kildal et al., "Threshold receiver model for throughput of wireless devices with MIMO and frequency diversity measured in reverberation chamber," IEEE Antennas Wireless Propag. Lett., vol. 10, pp. 1201-1204, 2011.

[2] P.-S. Kildal, C. Orlenius, and J. Carlsson, "OTA testing in multipath of antennas and wireless devices with MIMO and OFDM," Proc. IEEE, vol. 100, no. 7, pp. 2145-2157, Jul. 2012.

[3] X. Chen, P.-S. Kildal, and M. Gustafsson, "Characterization of implemented algorithm for MIMO spatial multiplexing in reverberation chamber," IEEE Trans. Antennas Propag., vol. 61, no. 8, pp. 4400-4404, Aug. 2013.

[4] N. Arsalane et al., "3GPP channel model emulation with analysis of MIMO-LTE performances in reverberation chamber," Int. J. Antennas Propag., vol. 2012, p. 239420, 2012.

[5] R. Recanatini, F. Moglie, and V. Mariani Primiani, "Performance and immunity evaluation of complete WLAN systems in a large reverberation chamber," IEEE Trans. Electromagn. Compat., vol. 55, no. 5, pp. 806-815, Oct. 2013.

[6] K. A. Remley, H. Fielitz, H. A. Shah, and C. L. Holloway, "Simulating MIMO techniques in a reverberation chamber," in Proc. IEEE EMC Symp., Aug. 2011, pp. 676-681.

[7] National Instruments, Austin, TX, USA, "NI USRP," [Online]. Available: http://www.ni.com/usrp/

[8] A. Paulraj, R. Nabar, and D. Gore, Introduction to Space-Time Wireless Communication. Cambridge, U.K.: Cambridge Univ. Press, 2003.

[9] National Instruments, Austin, TX, USA, "LabVIEW system design software," [Online]. Available: http://www.ni.com/labview/

[10] P.-S. Kildal, X. Chen, C. Orlenius, M. Franzen, and C. S. L. Patane, "Characterization of reverberation chambers for OTA measurements of wireless devices: Physical formulations of channel matrix and new uncertainty formula," IEEE Trans. Antennas Propag., vol. 60, no. 8, pp. 3875-3891, Aug. 2012.

[11] 3rd Generation Partnership Project, "LTE physical layer-General description," Tech. Rep. TS 36201, V8.1.0, Nov. 2007. 